\title{
LOS MARIDOS EN THE CANTERBURY TALES
}

\section{F. Javier Sánchez Escribano}

Antes de comenzar el estudio de los maridos en The Canterbury Tales es necesario que nos hagamos la pregunta de qué es el matrimonio en contraposición al amor ccrtés, el amante y su señora. Acosado por limitaciones de tiempo y espacio, mi estudio no puede ser más que una introducción a lo que pudiera constituir un trabajo mucho más amplio.

La mujer es un ser indefenso, unido a la tierra, con dos posibles finales: el matrimonio o el convento. Nos interesa el primero. Dice G.G. Coulton en Chaucer and His England que en el sistema feudal "land was necessarily held by personal service; therefore the woman, uselless in war, must necessarily be given with her land to some man able to defend it and her"1. Sistema, pues, que se apoya exclusivamente en el hombre. De ahí que la mujer vaya al matrimonio unida a unas tierras o a una dote, valorándose más aquélla cuanto más importantes sean las segundas. El matrimonio va unido a una conveniencia para la que la voluntad de la contrayente carece de importancia. Los matrimonios entre niños son corrientes, con la única limitación de la edad, 12 años para el hombre y 7 para la mujer, como mínimo. Con la misma facilidad que se llevan a cabo pueden destruirse, bastando para ello comprar a la Iglesia un libelo que declare nulo el matrimonio y envíe a la mujer de vuelta a la casa de su padre.

Al mismo tiempo y opuesto a la moral cristiana, el amor cortés desarrolla un sistema y una terminología derivados de ideas cristianas, en los que la exaltación de la mujer es su punto de partida, en conexión, quizá, con la reverencia prestada a la Virgen María. El paralelismo entre la religión de Cristo y la de $\mathrm{Cu}$ pido se ve en las expresiones comunes a ambas, como dice Nevill Coghill en The Poet Chaucer: "conversión, penance, service, prayer, fasting, martyrdom, 
sanctity, faith, works, and hope in the lover; in the lady, grace, pity, mercy,

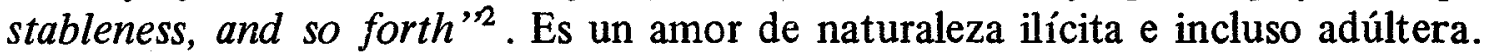
$\mathrm{Su}$ fin no es el matrimonio. El corazón del amante late por una amante no por el de una esposa. El matrimonio cristiano, sacramento que se toma con la idea de procreación de los hijos, es también un contrato en el que la mujer jura obediencia a su esposo y no puede abandonarle sin romper dicho juramento. El matrimonio destruiría el amor, puesto que invierte el orden de las cosas. El suplicante y siervo vendría a ser dueño y señor.

$\mathrm{El}$ amante debe ser fiel y secreto, teniendo en consideración su honor y el de su dama. Puede tener un solo amigo a quien confiará el secreto de su pasión. Debe ser generoso, valiente, compasivo, humilde y, sobre todo, constante y fiel, sin límite de edad. La dama, por su parte, "was a complex of yielding and withdrawal. She must above all be beautiful and chaste in heart, that is, not given to other men, nor indeed too easily to her chosen lover. The qualities of pity, welcomingness, and bounty of soul (what is called "Franchise") will move her towards the lover. But she must also be shamefast and guarded by a sense of Daunger (Disdain), and fear the loss of her reputation by the discovery of her lover. These will give her pause in yielding; but once yielded, constancy and faith is as much demanded of her as of her lover"' .

En The Canterbury Tales nos vamos a encontrar con estas ideas en continua oposición. Unas veces el adulterio estará justificado como castigo al inarido celoso e ignorante, otras será la lujuria de la esposa, cuando no, será la omnipotencia del marido. La lucha por el poder puede definir muy bien el estado del matrimonio en estos cuentos. Seguramente Chaucer se inclinará por una postura ecléctica, en la que no se hablará tanto de poder como de amor. Analicemos, pues, el comportamiento de los maridos en los cuentos que tienen una mayor relación con el tema que nos ocupa.

\section{Cuento del Molinero}

En la discusión del Hostelero y del Molinero que precede al cuento, este último, en medio de su borrachera, responde al Mayordomo que le ha advertido de que es un pecado calumniar a un hombre y traer escándalo sobre las esposas en general:

“...Leve brother Osewold,

Who had no wyf, he is no cokeold.

But I say nat therfore that thou art oon;

Ther been ful goode wyves many oon,

And evere a thousand goode ayeyns oon badde". 3151-54 .

2 Nevill Coghill: The Poet Chaucer. London, Oxford University Press, 1971, p. 14.

3 Ibídem, pp. 13-14.

4 Debemos advertir que todas las citas de este trabajo provienen de The Works of Geoffrey 


\section{LOS MARIDOS EN THE CANTERBURY TAIES}

Con ello el molinero introduce un tema que en su cuento va a ser el punto de partida y el de llegada de su protagonista: el marido cornudo. John es un viejo carpintero que ama a su esposa más que a su propia vida, pero que con sus celos y su desconfianza destruye la felicidad que hubiera podido obtener con una joven esposa de dieciocho años:

"Jalous he was, and heeld hire narwe in cage,

For she was wylde and yong and he was old,

And demed hymself been lik a cokewold". 3224-65 .

El problema de los celos del carpintero no proviene de ninguna falta de Alisoun, su esposa, sino por su propia obsesión en la diferencia de edad, pues él es viejo y ella joven; no que ella le haya engañado sino de verse desposeido de algo que sólo ella puede disfrutar. Los consejos de Catón, a este respecto, no pueden ser más significativos, al mismo tiempo que insiste en la incompatibilidad que existe entre la vejez y la juventud. Encerrando a su esposa "narwe in cäge" lo que pretende es controlar algo que no puede retener, es decir, la juventud con todas sus consecuencias. La trampa en la que ha caído es su propio matrimonio, como muy bien dice Trevor Whittock: "The snare into which he has fallen is marriage with a younger wife whom he loves, but again we can detect in the lines profounder implications: the snare is that of life itself, and the cares are those human troubles no one can escape"6. Por otra parte, encerrado en su propia ignorancia, cae en la trampa tendida por Nicolás, que se aprovecha de su superstición. Seguramente no le hubiera engañado si no se hubiera preocupado tan apasionadamente de su esposa, como nos lo demuestra con su "Allas, my wyf! And shall she drenche?" (vv. 3522-3), una vez que el estudiante le ha dado parte de su sueño y de la inminencia de un diluvio.

La ironía de este cuento debemos verla no sólo en el engaño del marido sino también en el de los amantes. Estos, afeminados y empleando un lenguaje propio del amor cortés, salen trasquilados. La derrota es tanto para la ignorancia del marido como para los superinteligentes mancebos, sobre todo la de Nicolás. Frente a éstas, Alisoun es como un regalo de Dios para bendición de los hombres. Es su juventud frente a la vejez de su marido, la tiranía frente a la existencia independiente. Es una suma de contrarios maravillosamente mezclados en ella, como dice T. Whittock: "The description... of her clothes displays

Ghaucer. Edited by F.N. Robinson. London, Oxford University Press, 1970. 1002 pp. Igualmente, la traducción de los cuentos la sacamos de la edición a cargo de Caridad Oriol, traducida por Juan Canti Bonastre. Editorial Bruguera, Barcelona, 1975 "Querido Oswald, cuando un hombre no tiene esposa no puede ser cornudo. No es que yo insinúe que tú lo eres. Hay montones de esposas fieles, un millar por cada una que no lo es". p. 103.

5 "Como era joven y caprichosa y él era viejo, los celos le inducian a tenerla siempre estrechamente vigilada y raramente la dejaba salir sula; y'a se consideraba a si mismo un posible cornudo". ibídem, p. 105.

6 Trevor Whittock: A Reading of the Canterbury Tales. Cambridge, At the University Press, 1968, p. 81 . 


\section{JAVIER SANCHEZ ESCRIBANO}

her sexual attractiveness, feminine pride, and healthy neatness. Various contraries are marvellously unified in her: wantonness and innocence, primness and fertility, softness and strength, tenderness and independence, haughtiness and playfulness, repose and spontaneity" . Y la única que sale bien parada es precisamente ella:

"Thus swyved was this carpenteris wyf,

For al his kepyng and his jalousye;

And Absolon hath kiss hir nether ye;

And Nicholas is scalded in the towte". $3850-3^{8}$.

\section{Cuento y Prólogo de la Mujer de Bath}

En este cuento Chaucer nos presenta a uno de los personajes cómicos más importantes de la Literatura Inglesa, sólo comparable a Falstaff, su contemporáneo, por otra parte. En su prólogo, Alisoun, que así se llama la mujer de Bath, nos hace la primera autobiografía en lengua inglesa. El tema más importante de su relato es la lucha de los sexos por "maiestrye" y "sovereignty", o "the eternal struggle between male and female, in which the one attempts to deny the challenge and being of the other by domination or agression" . Representa una figura matriarcal que ha declarado la guerra al hombre, la eterna revuelta femenina contra la civilización ordenada y centrada en el macho.

G. Rattray Taylor en Sex in History ${ }^{10}$ dedica un capítulo al estudio de la moral sexual medieval, dándonos a conocer las limitaciones que la propia Iglesia imponía al matrimonio, desde los libros de penitencia, en donde se describen las faltas y los castigos correspondientes a cada una de ellas, donde se ensalza la virginidad, hasta la prohibición del acto sexual en el quivalente de más de cinco meses al año. Para la Iglesia la mujer es un mal necesario, una tentación natural, una calamidad deseable, un peligro doméstico y una fascinación mortal. Y precisamente la sátira del Prólogo de la mujer de Bath va dirigida contra las actitudes obsesionadas por el sexo de la Cristiandad medieval.

Alma inquieta, con grandes conocimientos sagrados y profanos, la Mujer de Bath pone fuera de combate a cinco maridos, "the five martyrs" como los llama G.G. Coulton. Después de hablar de la Samaritana que, como ella, ha tenido cinco maridos, se compara con Salomón que tuvo innumerables mujeres.

$7 \quad$ Ibidem, p. 86.

8 "Asi fue cómo, a pesar de los celos y de la vigilancia, la mujer del carpintero burló a su marido; Absalón besó el culo de Eloisa y el de Nicolás resultó chamuscado". Caridad Oriol, op. cit., p. 119.

9 Trevor Whittock, op. cit. pp. 118-9.

10 G.R. Taylor, Sex in History. Thames and Hudson, 1953. Chap. III: "Medieval Sexual Morality", citado por Trevor Whittock, ibídem, pp. 119 a 121. 


\section{LOS MARIDOS EN THE CANTERBURY TALES}

Admite la autoridad de Cristo pero no la virginidad:

"Crist was a mayde, and shapen as a man, And many a seint. sith that the world began;

Yet lyved they evere in parfit chastitee.

I nyl envye no virginitee.

Lat hem be breed of pured whete-seed,

And lat us wyves hoten barly-breed". 139-144" .

Esta mujer es el elemento del que se sirve Chaucer para satirizar ambas actitudes, la del macho y la de la hembra. Ella misma es un grotesco ejemplar de muchos de los vicios femeninos, como dice T. Whittock: "nagging, scolding, deceiving, chiding, grumbling, spending, gossiping, lying and betraying. She is vain, egotistic, hypocritical (as when she attends the funeral of her forth husband), prossessive and licentuous"

De sus cinco maridos, tres fueron buenos y dos malos, basándose esta división en el grado de facilidad con que pudo dominarlos:

\section{"...tho housbondes that I hadde,}

As thre of hem were goode, and two were badde.

The thre were goode men, and riche, and olde;

Unne the myghte they the statut holde

In which that they were bounden unto me.

Ye woot wel what I meene of this, pardee!

As help me God, i laughe whan I thynke

How pitously a-nyght I made hem swynke!" 195-202 ${ }^{13}$.

Resulta obvio que su deseo es establecer la dominación femenina sobre la masculina. "Sovereignty" significa romper la voluntad masculina y poseerla copletamente, como ella misma nos relata:

An housbonde I wol have, I wol nat lette,

Which shal be bothe my dettour and my thral,

And have his tribulacioun withal

Upon his flessh, whyl that I am his wyf.

11 "Cristo era virgen, muchos de los santos también lo fueron, y a pesar de que sus cuerpos eran como los demás hombres, guardaron perfecta castidad. En este inmenso campo que es el mundo, dejad que las virgenes sean el trigo y nosotras, las esposas, la cebada". Caridad Oriol. op. cit., p. 258.

12 Trevor Whittock, op. cit. p. 124.

13 "De mis cinco maridos tres fueron muy fuenos, malos los otros dos. Los tres que he calificado como buenos eran ricos y decrépitos y a pesar de sus grandes esfuerzos ape. nas podian satisfacer el tributo exigido por el matrimonio. Ya imagináis, supongo, a lo que hago alusión. Aún me río cuando recuerdo de qué manera tan despiadada les hacia trabajar por las noches". Caridad Oriol, op. cit. p. 259. 


\section{JAVIER SANCHEZ ESCRIBANO}

I have the power durynge al my lyf

Upon his propre body, and noght he". 154-9 ${ }^{14}$.

El último marido, un joven estudiante de veinte años llamado Jankin, fue el peor de todos $\mathrm{y}$, sin embargo, al que más amaba. Fue el peor de todos porque también fue el único que le devolvió el golpe.

Curiosamente la moraleja de su cuento parece obvia: el Caballero consigue su recompensa después de ceder el poder a la mujer; pero, sin embargo, la verdadera moraleja debemos verla en el reconocimiento del valor real del otro. El Caballero viola a una mujer, lo que significa que la considera como atributo de su propia personalidad, como objeto que se puede violar por la fuerza. Como castigo la Reina le impone la solución de un acertijo antes de un año: debe saber qué es lo que más desean las mujeres. Por fin encuentra la respuesta en boca de una mujer vieja y fea, la cual le pide una promesa que, como caballero, deberá cumplir, a saber, concederle lo que ella le pida. La respuesta al acertijo es la siguiente:

"Wommen desiren have sovereynetee As wel over hir housbond as hir love, And for to been in maiestrie him above". 1038-1040 ${ }^{15}$.

Para cumplimiento de su promesa el Caballero debe casarse con la anciana, que nuevamente le pone a prueba: debe elegir entre que sea fea pero fiel o hermosa e infiel. El sabe dar la respuesta exacta: no toma ninguna de las dos alternativas sino que se subordina a su responsabilidad, dejando que ella elija lo que crea más conveniente para beneficio de ambos:

"Chese now", quod she, "oon of thise thynges tweye:

To han me foul and old til that I deye, And be to yow a trewe, humble wyf, And nevere yow displese in al my lyf;

Or elles ye wol han me yong and fair, And take youre aventure of the repair That shal be to youre hous by cause of me, Or in som oother place, may wel be".

"My lady and my love, and wyf so deere, I put me in youre wise governance; Cheseth yourself which may be moost plesance, And moost honour to yow and me also". 1219-33' ${ }^{16}$.

14 "Quiero un esposo que sea a la vez mi deudor y mi esclavo; mientras sea su mujer, tendrá su "tormento de la carne". Seré yo y no él, la que dominará las potencias de su cuerpo". Ibídem, p. 258.

15 "Lo que las mujeres desean con más vehemencia es tener autoridad sobre sus esposos y sus amantes"'p. 278.

16 "No obstante -continuó diciendo ella-, debes escoger entre dos cosas: aceptarme tal como soy por el resto de mis dias, pero fiel y obediente, o bien que sea joven y hermo- 


\section{LOS MARIDOS EN THE CANTERBURY TALES}

Con lo cual consigue que su esposa sea fiel y hermosa. Como recompensa obtiene la libre entrega de su esposa, como apunta T. Whittock: "By his full recognition of her "otherness" he is rewarded by her free acceptance of him. Thus, in the mutual recognition of the other, in each giving only to find that the giving is the taking, in this lies the ideal love-marriage relations" 17 , que viene a ser la verdadera moraleja de este cuento.

\section{Cuento del Estudiante}

Este cuento, que parece una respuesta al de la Mujer de Bath, nos enseña que el amor puede ser la abnegación de uno en favor del otro, la importancia de la disciplina, en suma, la virtud de la paciencia. Cuando su pueblo le pide a Walter que escoja una esposa, decide hacerlo pero con una condición: deben aceptar a la mujer que él elija. Su elección recae sobre Griselda, valiosa elección, si cabe, porque demuestra su perspicacia en la apreciación del verdadero valor de aquélla. Aunque parece hacerle un favor, hace que ella le prometa entregársele en hechos y pensamientos. En suma, el marido quiere tener la soberanía:

"I seye this, be ye redy with good herte

To al my lust, and that I frely may

As me best thynketk, do yow laughe or smerte, And nevere ye to grucche it, nyght ne day?

And eek whan I sey "ye", ne say nat "nay",

Neither by word ne frownyng contenance?

Swere this, and heere I swere oure alliance".

Wondrynge upon this word, quakynge for drede,

She seyde, "Lord, undigne and unworthy

Am I to thilke honour that ye me beede,

But as ye wol yourself, right so wol I.

And heere I swere that nevere willyngly,

In werk ne thought, I nyl yow disobeye,

For to be deed, though me were looth to deye". 351-364 ${ }^{18}$

sa y que todos los hombres vengan a tu casa para asediarme”... " jMi señora y mi amor, mi bien amada esposa! Pongo en ti toda mi confianza y quiero que seas tú misma la que decida, pues estoy seguro que escogerás lo que sea más placentero y honroso para ambos". Ibídem. p. 282.

17 Trevor Whittock, op. cit. p. 127.

18 "Si lo estás (decidida), ¿me prometes acceder a todos mis deseos, que seré libre de hacer lo que crea más conveniente, ya te haga feliz o te apesadumbre? ¿Que nunca te quejarás ni me contrariarás, que cuando yo diga "si" tú no dirás "no", ya sea de palabras o con un simple gesto? Prométemelo y te juro que serás mi esposa.

Aturdida por estas palabras, la joven replicó con temblorosa voz:

- Mi señor, no merezco el alto honor de ser vuestra esposa. Vuestros deseos serán ahora los mios. Bajo mi humilde techo os juro, que no seré obstinada y que os obede- 


\section{JAVIER SANCHEZ ESCRIBANO}

Sigue una descripción de la felicidad de este matrimonio. Pronto Walter decide poner a prueba el poder que tiene sobre su esposa y lo hace con una refinada crueldad. Le arrebata a sus hijos aludiendo a su bajo linaje con una patente poca sinceridad. El encargado de realizar este hecho cumple con su deber de la manera más cruel que puede. La humilla hasta en la manera de enviarla a su casa, tras de una supuesta compra de un libelo y la consecuente anulación del matrimonio. Ella, cuya imagen más próxima la vemos en Abraham, responde con su honestidad en cumplimiento de la palabra dada:

I have noght had no part of children tweyne

But first siknessa, and after wo and peyne.

"Ye been oure lord, dooth with youre owene thyng

Right as yow list; axeth no reed at me.

For as I lefte at hoom al my clothing,

Whan I first cam to yow, right so", quod she

"Lefte I my wyl and al my libertee,

And took youre clothing; wherfore I yow preye,

Dooth youre plesaunce, I wol youre lust obeye". 650-8 ${ }^{19}$.

Con estas humillaciones Walter nos demuestra que acepta el matrimonio como "maiestrye" del marido. Abusa de su autoridad como esposo y explota las obligaciones de sumisión que, hechas, hacen de la esposa la esclava de un señor feudal representado por el esposo. Es Griselda la que nos da una lección moral en este caso, al amar a su esposo por lo que vale y a pesar suyo. Su amor al hombre va unido a su deber de sumisión al esposo y señor feudal. Es raro encontrar una Griselda por el mundo. Por eso Chaucer da un consejo a los maridos: no abuséis de la paciencia de vuestras mujeres:

"Griselde is deed, and eek hire pacience, And bothe atones buryed in Ytaille;

For which I crie in open audience, No wedded man so hardy be t'assaille

His wyves pacience in trust to fynde

Grisildis, for in certain he shal faille". 1177-82 ${ }^{20}$.

ceré en todo, aunque ello sea la causa de mi muerte $y$, os advierto, con toda sinceridad, que no quiero morir". Caridad Oriol, op. cit. p. 319.

19 "Apenas pude gozar de ellos a no ser en tiempo de enfermedad o de dolor. Tú eres nuestro dueño y señor, haz lo que creas más conveniente con lo que te pertenece, prescinde de mis consejos. Asi como dejé mis pobres vestidos en casa para casarme contigo, de la misma manera dejé alli abandonadas mi libertad y mi voluntad para sujetarme a tus deseos y obedecerte en todo". Ibídem. p. 325.

20 "Griselda murió, murió su paciencia, las dos descansan en el mismo lugar; a vosotros, maridos, recomiendo prudencia, no os arriesguéis en querer probar a vuestras esposas, como el marqués a Griselda, ya que en el intento podéis muy bien fracasar". Ibídem, p. 337. 


\section{LOS MARIDOS EN THE CANTERBURY TALES}

She seith nat ones "nay", whan he seith "ye".

"Do this", seith he; "Al redy, sure", seith she. 1337-46"2.

No aceptará ni a una mujer mayor ni a una viuda, puesto que sabrían más que él. Prefiere una de veinte años para moldearla a su gusto. De sus consejeros, Placebo es la imagen perfecta, al mismo tiempo que una sátira, del adulador de una corte, que sigue la corriente de la vanidad de su amigo. Justino, por su parte, muestra más sentido común y reconoce que una esposa requiere incluso más atención que la entrega de una propiedad, puesto que lo que en el matrimonio se entrega es el propio cuerpo $\mathrm{y}$, lo más probable, es que un hombre viejo comó él no pueda satisfacer muchas de las demandas que una mujer joven le pueda hacer.

En la descripción de la boda y en lo que sigue, donde se nos muestra toda la lujuria del anciano, vemos una grotesca parodia del significado del matrimonio. Vemos crecer el deseo del viejo en medio de la borrachera de la fiesta nupcial, su preparación para hacer el amor tomando afrodisíacos, en suma, vemos su respulsiva apariencia física. Curiosamente, no se nos dice cuál es el sentimiento de la esposa, aunque podemos adivinar el horror de su experiencia. El humor lo vemos en que el esposo cree ser el más fuerte sexualmente, cuando en realidad es ella la que encierra toda la vitalidad sexual. Con la entrada de Damián, la esposa comienza el aprendizaje. Pero January queda ciego y no deja a May fuera de su alcance. Su ceguera significa en él una falta total del conocimiento de sí mismo y de sus celos. Su inocente esposa se ha transformado en una persona dura y calculadora. Por fin, ambos se encuentran a la misma altura pues la reconoce como una persona de derecho, como muy bien apunta Trevor Whittock: "Where at first he thought of her only as an instrument for his own gratification there comes gradually a recognition of her as a person in her own right, and even a degree of love",23 , cuando dice:

"Now wyf", quod he, "heere nys but thou and I,

That art the creature that I best love.

For by that Lord that sit in hevene above,

Levere ich hadde to dyen on a knyff,

Than thee offende, trewe deere wyf!" $2160-4^{24}$.

22 “'Tener una esposa! ¿Qué mal le puede acontecer a un hombre por tomar esposa? ninguno, creo yo. No hay palabras para describir la felicidad de aquellos dos seres. Si el marido es pobre, le ayuda ella en el trabajo, velando con todo interés por las cosas del hogar sin malgastar un penique; lo que al esposo le agrada, a ella le deleita; cuando él dice "si", a ella no se le ocurre decir "no". Si él dice: "Haz esto", ella contesta: "Al momento querido". Ibídem. p. 343.

23 Trevor Whittock op. cit., p. 159.

24 "Estamos ahora completamente solos los dos. Pongo al cielo por testigo, que antes hundiría una daga en mi corazón que causarte el más leve daño". Caridad Oriol, op. cit. p. 360. 


\section{JAVIER SANCHEZ ESCRIBANO}

De esta manera, condenando la conducta de Walter, este cuento refuerza el ataque de la Mujer de Bath contra el matrimonio cristiano por dar demasiado poder al marido.

\section{Cuento del Mercader}

Nuevamente nos encontramos con la guerra entre los sexos, pero esta vez en una posición media: ambos abusan el uno del otro. Se repite el tema de la incompatibilidad de la vejez y de la juventud, lo mismo que la primavera se opone al invierno, representados no sólo por May (Mayo) y January (Enero), respectivamente, sino también por Proserpina y Plutón.

January es un mercader de sesenta años que, después de una vida de deleites con las mujeres, decide buscar esposa. La primera parte del cuento está dedicada a presentarnos la ignorancia del Mercader en lo que al matrimonio se refiere. Su teoría no es más que una ilusión, una ceguera de lo que ocurre a su alrededor. De nuevo la imagen de la mujer como objeto para procreación de los hijos aparece en escena.

"And certeinly, as sooth as God is kyng,

To take a wyf it is a glorious thyng,

And namely whan a man is oold and hoor;

Thanne is a wyf the fruyt of his tresor.

Thanne sholde he take a yong wyf and feir,

On which he myghte engendren hym an heir,

And lede his lyf in joye and in solas". 1267-73 21 .

al mismo tiempo que una falsa imagen de lo que es la vida de matrimonio, siempre según el viejo mercader:

A wyf! a, Seinte Marie, benedicitee!

How myghte a man han any adversitee

That hath a wyf? Certes, I kan nat seye.

The blisse which that is bitwixe hem tweye

Ther may no tongue telle, or herte thynke.

If he be povre, she helpeth hym to swynke;

She kepeth his good, and wasteth never a deel;

Al that hire housbonde lust, hire liketh weel;

21 "Tan cierto es como la existencia de Dios, que excelente estado es el del matrimonio, especialmente cuando el novio es viejo y con achaques, pues entonces la mujer se convierte en su más preciada joya. Sin embargo, el caballero estaba decidido a escoger para esposa a una mujer joven y bella y de esta manera asegurar la descendencia, disfrutando, además, de una atrayente compañia en el lecho". Ibídem, p. 341. 


\section{LOS MARIDOS EN THE CANTERBURY TALES}

And nat to been constreyned as a thral;

And so doon men, if sooth seyen shal. $764-70^{26}$.

Después unifica el ideal del matrimonio cristiano y la tradición cortés del amor romántico, deber y deleite unidos en un todo:

Thus hath she take hir servant and hir lord,

Servant in love and lord in marriage.

Thanne was he both in lordshipe and servage.

Servage? nay, but bothe his lady and his love:

His lady, certes, and his wyf also,

The which that lawe of love acordeth to. $792-8^{27}$.

$\mathrm{Y}$ comienza el cuento propiamente dicho. Arveragus debe ausentarse por un año o dos. Tema de la ausencia del marido que vuelve a repetirse. Dorigen, por su parte, se ve acosada por una extraña preocupación simbolizada en las rocas de los acantiliados, miedo por la salvación de su marido y obsesión por un desastre, sentimientos que nos parecen excesivos. Quizá lo que más le preocupa a ella son sus propias pasiones ocultas que no le dejan disfrutar de la belleza y la armonía del jardín y de la propia vida. Llevada por esa obsesión contrae otra promesa, ésta con Aurelio, de ser suya cuando haga desaparecer los acantilados, cosa que consigue con la ayuda de un mago. El mundo de la ilusión vuelve a hacer su aparición, puesto que los acantilados no desaparecen sino que es una imagen que les impone el mago. La condición se ha cumplido, ahora es la esposa la que debe cumplir su promesa. Cuando cuenta a Arveragus lo ocurrido, lo toma con una entereza extraordinaria y no rehuye la realidad. Por el contrario, él responde con calma:

"Is ther oght elles, Dorigen, but this?"

"Nay, nay", quod she, "God helpe me so as wys!

This is to muche, and it were Goddes wille".

"Ye, wyf", quod he, "lat slepen that is stille.

It may wel, peraventure, yet to day.

Ye shul youre trouthe holden, by my fay!

For God so wisly have mercy upon me,

I hadde wel levere ystiked for to be

For verray love wich that I to yow have,

But if ye sholde youre trouthe kepe save.

26 "El amor no debe ser forzado ni limitado por el dominio, ya que cuanto éste aparece, el dios encoge sus alas y emprende la retirada. Al amor no se le pueden señalar fronteras. Las mujeres por propia naturaleza desean la libertad no quieren ser tratadas como esclavas y lo mismo sucede con los hombres, si no me equivoco". Ibídem. p. 384.

27 "Ella le tomó por su siervo y señor, en el amor su siervo, en el matrimonio su dueño. De este modo quedó el caballero convertido en ambas cosas. No, siervo no lo era, pues era el dueño de su dama y de su amor. Era su dama ciertamente. pero también su esposa ente Dios y ante los hombres ${ }^{2}$. Dbídem. p. 384. 


\section{JAVIER SANCHEZ ESCRIBANO}

El desenlace final tiene lugar en el jardín, que representa tanto al de $L e$ Roman de la Rose como al del matrimonio. Al mismo tiempo que January pretende establecer su dominio sobre May, ésta prepara su engaño, y lo mismo ocurre entre Plutón y Proserpina. El viejo recupera la vista y la esposa encuentra una respuesta aparente. Y la ceguera, la ilusión, sigue porque él queda feliz después de las explicaciones de la esposa y termina por no apercibirse de la mala conducta de ésta.

\section{Cuento del Terrateniente}

El cuento de Arveragus, Dorigen y Aurelio, recopila incidentes y temas ya expuestos en cuentos anteriores, como son: un inexplicable sufrimiento (el de Dorigen ante la ausencia de su esposo), los valores que deben presidir la vida del hombre, atenerse a la verdad y vivir con ella. Curiosamente es el propio Terrateniente el que, al narrar su cuento, da las principales normas que deben regir al amor.

El amor cortés preside la declaración amorosa, el propio contrato matrimonial e, igualmente, la declaración amorosa del amante Aurelio. Dorigen se apiada de las penas y desesperación de aquél, como ya lo hiciera de las de Arveragus, al que acepta como esposo y señor "Of swich lord shipe as men han over hir wyves" (v. 743). El, por su parte, para más alegría de sus vidas

Of his free wyl he swoor hire as a knyght

That nebere in al his lyf he, day ne nyght

Ne sholde upon hym take no maiestrie

Agayn hir wyl, ne kithe hire jalousie,

But hire obeye, and folwe hir will in al,

As any lovere to his lady shal,

Save that the name of soveraynette,

That wolde he have for shame of his degree. 745-52 ${ }^{25}$.

Como decíamos, es el mismo narrador quien nos dice las palabras más sabias respecto al matrimonio. Primero rechaza la "maiestrie" porque aleja al amor:

Love wol nat been constreyned by maiestrye.

Whan maistrie comth, the God of Love anon

Beteth his wynges, and farwel, he is gon!

Love is a thing as any spirit free.

Wommen, of kynde, desiren libertee,

25 "El caballero juró sobre su espada que mientras viviera jamás ejercitaría su autoridad sobre su esposa, ni se dejaría arrebatar por los celos, sino que en todo momento accederia a sus deseos, como acostumbra ha hacerlo todo enamorado con su dama; pero que para no menoscabar su honor de caballero, externamente darian la impresión de que asumia todos sus derechos como marido". Ibídem, p. 383. 


\section{LOS MARIDOS EN THE CANTERBURY TALES}

Trouthe is the hyeste thyng that man may kepe". 1469-7928.

Debemos atenernos a la verdad y cumplir la promesa hecha. La misma "gentillesse" del marido la vemos en Aurelio, que libra a Dorigen de la palabra dada, y en el mago, que perdona a Aurelio las mil libras que costaba el hechizo. El marido se impone con la verdad, su generosidad y su "gentillesse".

\section{Cuento del Marino}

De nuevo es un mercader la víctima de un cuento de Chaucer. El triángulo, cuyos vértices son marido, esposa y amante, vuelve a repetirse, pero esta vez aparece un nuevo elemento, el dinero, con todos sus peligros, como nos apunta al comienzo del cuento:

The sely housbonde, algate he moot paye,

He moot us clothe, and he moot us arraye,

Al for his owene worshipe richely,

In which array we daunce jolily.

And if that he noght may, por aventure,

Or ellis list no swhich dispence endure,

But thynketh it is wasted and ylost,

Thanne moot another payen for oure cost,

Or lene us gold, and that is perilous. 11-1929.

A primera vista parece que nos encontramos ante un esquema muy común según el cual un amante joven y viril y una esposa hermosa y ligera, engañan a un marido ausente. Sin embargo vemos aparecer un cuarto elemento, como ya hemos anunciado, que es el dinero, verdadero motor de la acción. El marido, en este caso, es generoso con su esposa, al mismo tiempo que rico y preocupado por sus negocios. La esposa siente una preocupación desmesurada por el bien vestir, mientras que el amante, un monje bien puesto llamado Sir John, disfruta de la confianza de todos, incluida la de su convento, y de un apreciable bienestar. La esposa pide un préstamo al monje, más como un pretexto para gozar con su persona que por una necesidad de dinero. El monje, a su vez, se lo pide al

$28-i$ Es sólo eso, Dorigen?

- jOh, sí, sí! -gimió ella-. ¡Te lo juro! ¡Demasiado es, aunque sea la voluntad del Señor!

-Esposa mia - dijo Arverago--, deja de atormentarte, quizá todo saldrá bien. Harás honor a la palabra dada te lo juro. Antes quisiera que una espada enemiga me causara la muerte, que saber que has faltado a una promesa, pues nada hay más sagrado en el mundo que su cumplimiento". Ibídem. p. 399.

29 "Es siempre el marido el que paga; para mantener su prestigio, debe hacer lo imposible para que la esposa vista con elegancia y lleve caras joyas, y si no puede o no quiere gastar tanto dinero por considerar que es innecesario, entonces, algún otro prójimo debe hacerse cargo de la cuenta o bien prestar su importe a la dama, y aqui es donde realmente existe el peligro". Ibídem: p. 165. 


\section{JAVIER SANCHEZ ESCRIBANO}

marido $\mathrm{y}$, al poco tiempo, éste se ausenta de casa, ocasión que aprovechan los amantes para solazarse en el lecho matrimonial. Cuando vuelve de Brujas el marido necesita dinero y se lo pide a Sir John. Y éste le dice que ya se lo ha devuelto a la esposa.

El esquema de venta de la mujer lo vemos en un doble plano. Por un parte, ella no es que venda al monje sino que, simplemente, piensa que el dinero y el favor sexual son intercambiables. Por otra parte, hará lo mismo cuando el marido le reprenda por no haberle dado cuenta de la devolución del préstamo. En vez de con dinero lo pagará con otra moneda, en la cama:

"I wol answere yow shortly to the poynt.

Ye han mo slakkere dettours than am I!

For I wol paye yow wel and redily

Fro day to day, and if so be i faille,

Iam youre wyf; score it upon my taille,

And I shal paye as soone as ever I may.

For by my trouthe, I have on myn array,

And nat on wast, bistowed it so weel

For youre honour, for Goddes sake, I seye,

As be nat wrooth, but lat us laughe and pleye.

Ye shal my joly body have to wedde. $412-23^{30}$.

Con lo que el marido se ve más que complacido, seguramente porque puede jugar en casa a lo que no se atreve fuera. El dinero y el sexo se complementan en este cuento. De esta manera nadie sale perdiendo, como dice Trevor Whittock: "In this particular sophisticated comedy of adultery no harm has been done. The monk is not out of pocket, as the husband paid for his adventure; the husband does not lose, because his money stays with his wife (to whom he probably would have given it anyway) and for being cuckolded he is rewarded with his wife's greater amorousness; the wife gets the money, enjoys a lover and has her husband's spousal attentions",31.

\section{Cuento del Administrador}

Volvemos a encontrarnos con el tema del adulterio. Apolo tiene una esposa a la que ama más que a su propia vida. Sin embargo es tremendamente celoso. De nuevo vemos los consejos que leíamos en los cuentos del Molinero y del

30 "Pero como me hallo en una situación embarazosa y seguramente tienes deudores peores que yo, te propongo un trato: iré saldando la deuda poco a poco y ni no lo hago, por algo soy tu esposa, jcárgalo en cuenta! Te pagaré tan pronto como pueda. Te aseguro que no he derrochado el dinero sino que lo he empleado en vestidos, por lo tanto, no estés enfadado conmigo y seamos felices. En garantía te ofrezco mi lindo cuerpo". Ibídem. p. 174.

31 Trevor Whittock, op. cit. p. 200-1. 


\section{LOS MARIDOS EN THE CANTERBURY TALES}

Mercader, según los cuales no hay que perder el tiempo probando a las mujeres, puesto que, por otra parte, ningún poder puede detener algo que la naturaleza ha puesto en sus criaturas, es decir, el instinto. Su esposa le engaña durante su ausencia y fue testigo un cuervo al que había enseñado a hablar. Cuando éste le cuenta lo ocurrido su primera reacción es matar a su esposa para luego arrepentirse por creerla inocente, al mismo tiempo que castiga al cuervo.

La moraleja no es otra que aconsejar tener la boca cerrada y nunca decir a un marido celoso que su mujer le engaña:

Ne tellth nevere no man in youre lyf

How that another man hath dight his wyf;

He wol yow haten mortally, certein. 311-3 32 .

Así las cosas, podemos concluir que Chaucer arremete contra el señor feudal representado en sus cuentos por los maridos. Ridiculiza al "senex amans" haciéndolo ignorante o ciego. Son una diatriba contra las ausencias del marido mientras se tiraniza con los celos a la mujer, ocasión que aprovecha ésta para engañarle, sirviéndose, incluso, de su propio dinero. Igualmente arremete contra el marido celoso como otra muestra de tiranía. Su teoría del matrimonio parece estar en un término medio entre la de la Mujer de Bath y la de Walter en el cuento del Estudiante. La suya es, pues, una postura ecléctica donde no se hablará tanto de poder por el matrimonio como de amor.

32 "Jamás digáis a un hombre que otro se acostó con su esposa, pues sólo lograréis su enemistad y su odio". Caridad Oriol, op. cit. p. 442. 


\section{BIBLIOGRAFIA}

G.G. Coulton: Chaucer and his Englanc. London, Methuen and Co. Ltd. 1970. 283 págs.

N. Coghill: The Poet Chaucer. London, Oxfor University Press, 1971.145 págs.

G.R. Crampton: The Conditions of Creatures. New Haven and London, Yale University Press, 1974. 207 págs.

M. Hussey, A.C. Spearing, J. Winny: An Introduction to Chaucer. Cambridge, At the University Press, 1968. 192 págs.

Caridad Oriol, ed. por: Cuentos de Canterbury. Tra. por Juan Canti Bonastre. Barcelona, Editorial Bruguera, 1975. 556 págs.

F.N. Robinson, Edt. by: The Works of Geoffrey Chaucer. London, Oxford University Press, 1970. 1002 págs.

T. Whittock: A Reading of the Canterbury Tales. Cambridge, At the University Press, 1968. 309 págs. 